\title{
Dinámica folicular y relación cortisol-respuesta ovulatoria en vacas tratadas con diferentes cantidades de progesterona
}

\author{
González, S.A. ${ }^{2}$; Cabodevila, J.A.'; Callejas, S.S. \\ ${ }^{1}$ Fac. Cs. Vet. Univ. Nac. Centro Prov.Bs. As., Tandil. \\ Centro Investig. Vet. Tandil (CONICET-CICPBA), Argentina. \\ ${ }^{2}$ Actividad privada, Tandil. E-mail: callejas $($ vet.unicen.edu.ar
}

\begin{abstract}
Resumen
González, S.A.; Cabodevila, J.A.; Callejas, S.S.: Dinámica folicular y relación cortisolrespuesta ovulatoria en vacas tratadas con diferentes cantidades de progesterona. Rev. Vet. 31: 2, 109-114, 2020. El objetivo del presente estudio fue evaluar si el tratamiento con dispositivos intravaginales (DIV) con diferentes cantidades de progesterona (P4) afectaba la concentración plasmática de dicha hormona, la dinámica folicular y el porcentaje de ovulación en vacas. Además, se fijó como objetivo secundario ver si existía relación entre el nivel plasmático de cortisol y la respuesta ovulatoria. Vacas secas, cíclicas, Bos taurus, fueron asignadas aleatoriamente a 3 grupos que recibieron los siguientes tratamientos: 1) T0,558 $(\mathrm{n}=10)$, en el día 0 se colocó un DIV con $0,558 \mathrm{~g}$ de progesterona más benzoato de estradiol (BE). En el día 8 se retiró el dispositivo y se administró D-cloprostenol. En el día 9 se administró BE. 2) Tl $(n=10)$ y Tl,3 $(n=10)$, similares al grupo T0,558, con la salvedad que se utilizó un DIV con $1 \mathrm{~g}$ y $1,3 \mathrm{~g}$ de P4, respectivamente. No se observaron efectos del tratamiento sobre el porcentaje de ovulación (66,7\%), diámetro del folículo dominante ovulatorio y niveles de P4 plasmática. La concentración de cortisol en plasma fue mayor en las vacas que no ovularon. Se concluye que la cantidad de P4 contenida en los DIV evaluados no afecta la concentración de dicha hormona en plasma ni la dinámica folicular. El nivel de cortisol se relaciona con la respuesta ovulatoria y éste podría ser un indicador de estrés, el cual podría afectar la eficiencia reproductiva.
\end{abstract}

Palabras clave: vacas, progesterona, dispositivo intravaginal, cortisol, ovulación.

\begin{abstract}
González, S.A.; Cabodevila, J.A.; Callejas, S.S.: Follicular dynamic and relation cortisol-ovulatory response in cows treated with progesterone. Rev. Vet. 31: 2, 109-114, 2020. The objetives of the study were to evaluate whether treatment with intravaginal device (IVD) with different amount of progesterone (P4) affect P4 plasmatic concentration, follicular dynamic and ovulation rate; also, to determe the relationship between cortisol plasma level and ovulatory response. Non-suckling, cycling B. taurus beef cows were randomly allocated to groups: T0.558 $(\mathrm{n}=10)$ on day 0 , IVD with $0.558 \mathrm{~g}$ of $\mathrm{P} 4$ was inserted and oestradiol benzoate (OEB) was administered. On day 8, IVD removal and D-cloprostenol administration. On day 9, OEB administration; T1 (n=10), same protocol but an IVD with $1 \mathrm{~g}$ of P4; T1.3 $(n=10)$ : same protocol but an IVD with $1.3 \mathrm{~g}$ of $\mathrm{P} 4$. The ovulation rate $(66.7 \%)$, diameter of dominant follicle and $\mathrm{P} 4$ levels were not different among treatments $(\mathrm{p}>0.05)$. Cortisol levels were higher in cows that not ovulated on days 8 and $9(\mathrm{p}<0.05)$. In conclusion, IVD with $0.558,1$ and $1.3 \mathrm{~g}$ produce similar P4 levels and ovarian changes. Cortisol levels were higher in animals that did not ovulate, this being a possible indicator of stress, which can affect reproductive efficiency.
\end{abstract}

Key words: cows, progesterone, intravaginal device, cortisol, ovulation.

\section{INTRODUCCIÓN}

Diferentes tratamientos han sido utilizados para sincronizar los celos; la mayoría de ellos incluyen la administración de progesterona en combinación con un agente luteolítico y sales de estradiol, que permi-

Recibido: junio 2020 / Aceptado: septiembre 2020 ten regular la dinámica folicular y el momento en que ocurren las ovulaciones ${ }^{14}$. A este tratamiento de sincronización se lo utiliza en animales cíclicos ${ }^{6}$, como también en animales en anestro ${ }^{9}$.

En el mercado existen dispositivos que contienen diferentes cantidades de progesterona ${ }^{3}$. Así, se diseñó un experimento para caracterizar los niveles plasmáticos de progesterona utilizando el Cue-Mate $(1,56 \mathrm{~g}$ pro- 
gesterona, Bioniche Animal Health Canada Inc), CIDR (1,9 o 1,38 g progesterona; Pfizer Animal Health, USA) o DIB (1 g progesterona, Syntex SA, Argentina).

No se observaron diferencias en las concentraciones plasmáticas de dicha hormo-na durante un tratamiento de 7 días de duración. No obstante, el tratamiento con DIB o $1,38 \mathrm{~g}$ de progesterona generó el nivel mas bajo de progesterona plasmática en un período de 21 días, lo cual indica que los dispositivos con mayor cantidad de progesterona liberan dicha hormona durante un lapso de tiempo mas prolongado ${ }^{22}$

En Argentina y Brasil, existen dispositivos con contenidos de progesterona inferiores a $1 \mathrm{~g}$. En consecuencia, el presente trabajo tuvo por objetivo comparar el efecto de dispositivos con diferentes cantidades de progesterona sobre el nivel plasmático de dicha hormona, la dinámica folicular y la tasa de ovulación.

La administración de benzoato de estradiol al finalizar el tratamiento con progesterona podría inducir estro sin ovulación; a esto se lo ha asociado con la presencia de folículos pequeños ${ }^{6}$. Sin embargo, en otros casos, el estrés de los animales que se podría generar por las prácticas de manejo a la que son sometidos podría ser la causa de falta de respuesta ovulatoria.

Así, investigadores observaron que vacas que fueron manejadas en condiciones de estres tuvieron un menor porcentaje de preñez $(38,2 \%$ vs $63,1 \%$, respectivamente) ${ }^{27}$. En consecuencia, se fijó como objetivo secundario relacionar los niveles plasmáticos de cortisol con la respuesta ovulatoria.

\section{MATERIAL Y MÉTODOS}

Lugar, animales y tratamientos. El experimento se llevó a cabo en un establecimiento agropecuario ubicado en el partido de Rauch, Buenos Aires $\left(36^{\circ} \mathrm{S}, 59^{\circ}\right.$ W). Se utilizaron 30 vacas secas, cíclicas Bos taurus, que se ubicaron sobre pasturas naturales. La condición corporal al inicio del experimento fue de $3,4 \pm 0,4$ (escala 1 a 5 ; 1 : emaciada y 5 : obesa ${ }^{13}$. Los animales fueron distribuidos aleatoriamente a tres grupos que recibieron los siguientes tratamientos:

T0,558 $(\mathrm{n}=10)$. En el día 0 se colocó un dispositivo intravaginal con $0,558 \mathrm{~g}$ de progesterona (Cronipres M24, Biogénesis-Bagó, Garin, Argentina) y $2 \mathrm{mg}$ de benzoato de estradiol (BE, Bioestrogen, BiogénesisBagó, Garin, Argentina) administrado intramuscularmente (i.m.). En el día 8 se retiró el dispositivo y se administró vía IM $0,150 \mathrm{mg}$ de D-cloprostenol (Enzaprost, Biogénesis-Bagó, Garin, Argentina). En el día 9 (24 h post retiro del dispositivo), se inyectó $1 \mathrm{mg}$ de BE.

$T 1(\mathrm{n}=10)$. Ídem tratamiento $\mathrm{T} 0,558 \mathrm{con}$ la salvedad que la cantidad de progesterona fue de $1 \mathrm{~g}$ (Cronipres 3 usos, Biogénesis-Bagó, Garin, Argentina).

$T 1,3(\mathrm{n}=10)$. Ídem tratamiento $\mathrm{T} 0,558$ con la salvedad de que la cantidad de progesterona fue de $1,3 \mathrm{~g}$ (Cronipres 3 usos +3 camisas con $100 \mathrm{mg}$ de progesterona cada una, Biogénesis-Bagó, Garin, Argentina).
Toma de muestras y mediciones hormonales. Para caracterizar los niveles de progesterona y cortisol en plasmas se tomaron muestras de sangre por punción de la vena y ugular en los días $0,2,4,6,7,8,9,10,11$ y 22 . Dichas muestras se recogieron en tubos heparinizados y fueron centrifugados $(2000 \times \mathrm{g}$ durante 15 minutos) dentro de la hora de extraídas. El plasma sobrenadante fue recogido y almacenado a $-20^{\circ} \mathrm{C}$ hasta el momento de realizar las determinaciones hormonales.

Las mediciones hormonales se realizaron en el Laboratorio de Endocrinología de la Facultad de Ciencias Veterinarias (UNCPGA, Tandil, Buenos Aires). Las muestras se analizaron utilizando un kit de RIA para progeterona y cortisol (Coat Acount, Siemens Healthcare Diagnostic, USA). Los kits para progesterona ${ }^{25} y$ cortisol ${ }^{7}$ fueron previamente validados para su uso con plasma bovino.

Para progesterona, el coeficiente de variación intraensayo fue menor que $7 \%$ para concentraciones de $0,1 \mathrm{y}$ $40 \mathrm{ng} / \mathrm{ml}$; mientras que el coeficiente inter-ensayo tuvo una variación menor al 3,5\% y la sensibilidad del ensayo fue de $0,01 \mathrm{ng} / \mathrm{ml}$.

Para cortisol, el coeficiente de variación intra-ensayo fue menor que $9 \%$ para concentraciones entre 1 y $50 \mathrm{mcg} / \mathrm{dl}$, mientras que el coeficiente inter-ensayo fue menor que $5 \%$ y la sensibilidad fue de $1 \mathrm{mcg} / \mathrm{dl}$.

Mediciones ultrasonográficas. Se revisaron los ovarios mediante un scaner de 7,5 MGHz (Sonovet 900; Medison, Korea). Se determinó el área y la localización del cuerpo lúteo; como también, el diámetro de los folículos $>3 \mathrm{~mm}$. Se realizaron ecografías cada 48 $\mathrm{h}$ entre los días 0 y 6 ; cada $24 \mathrm{~h}$ del día 6 al 9 y cada 12 h del día 9 al 12. En el día 22 se realizó una nueva ecografía.

Las mediciones descriptas precedentemente permitieron determinar la dinámica folicular ocurrida durante el tratamiento, el área del cuerpo lúteo y el momento de la ovulación.

El día de emergencia de una nueva onda de crecimiento folicular fue definido como el momento en que el folículo dominante fue identificado retrospectivamente con un diámetro de $405 \mathrm{~mm}{ }^{10}$. Cuando el primer folículo identificado tuvo un diámetro de $6 \mathrm{~mm}$ se consideró al día previo como día de la emergencia folicular.

El folículo dominante de una onda de crecimiento folicular fue definido como el primer folículo que alcanzó el tamaño más grande ${ }^{11}$. En el presente estudio, el folículo dominante perteneciente a una nueva onda de crecimiento folicular fue inducido por el tratamiento con progesterona más estrógenos. Un folículo persistente fue definido cuando la dominancia de dicho folículo se extendió desde el momento de colocar el dispositivo intravaginal hasta el momento de la ovulación.

La tasa de crecimiento $(\mathrm{mm} /$ día) fue determinada en forma individual para cada uno de los folículos, utilizando el día como variable independiente y el diámetro como variable dependiente. Así, la tasa media de 
Tabla 1. Características foliculares en vacas secas tratadas con un dispositivo intravaginal con diferentes cantidades de progesterona (media $\pm E E$ ) para sincronizar la ovulación.

\begin{tabular}{|c|c|c|c|}
\hline \multirow{2}{*}{ variables } & \multicolumn{3}{|c|}{ cantidad de progesterona* } \\
\hline & $0,558 \mathrm{~g}$ & $1 \mathrm{~g}$ & $1,3 \mathrm{~g}$ \\
\hline FD al retirar el dispositivo intra-vaginal (mm) & $\begin{array}{c}10,7 \pm 0,6 \\
(8-15) \\
n=10\end{array}$ & $\begin{array}{c}11,0 \pm 0,5 \\
(9-12,5) \\
n=10\end{array}$ & $\begin{array}{c}10,9 \pm 0,4 \\
(9,5-13) \\
n=10\end{array}$ \\
\hline FD en el momento de administrar BE (mm) & $\begin{array}{l}11,8 \pm 0,9 \\
(8,5-16) \\
\end{array}$ & $\begin{array}{c}11,3 \pm 0,8 \\
(8-16) \\
\end{array}$ & $\begin{array}{c}11,2 \pm 0,6 \\
(8-14)\end{array}$ \\
\hline Porcentaje de ovulacion $(\%)$ & $60(6 / 10)$ & $70(7 / 10)$ & $70(7 / 10)$ \\
\hline Diámetro del FD ovulatorio (mm) & $\begin{array}{c}12,0 \pm 0,7 \\
(10-14) \\
n=6\end{array}$ & $\begin{array}{c}12,6 \pm 0,4 \\
(11,5-14) \\
n=7\end{array}$ & $\begin{array}{c}13,1 \pm 0,8 \\
(10-15) \\
n=7\end{array}$ \\
\hline Tasa de crecimiento ( $\mathrm{mm} /$ day) & $\begin{array}{l}0,7 a \pm 0,3 \\
(0,3-0,9) \\
\end{array}$ & $\begin{array}{l}1,1 b \pm 0,4 \\
(0,9-1,5)\end{array}$ & $\begin{array}{l}1,2 \mathrm{~b} \pm 0,4 \\
(0,7-1,6) \\
\end{array}$ \\
\hline Porcentaje de folículos persistentes & $30(3 / 10)$ & $10(1 / 10)$ & $10(1 / 10)$ \\
\hline Porcentaje de ovulación de los folículos persistentes (\%) & $100(3 / 3)$ & $0(0 / 1)$ & $0(0 / 1)$ \\
\hline
\end{tabular}

FD: foliculo dominante. BE: benzoato de estradiol. *Promedio con diferentes superindices en la misma fila difieren signiflcativamente $(p<0,05)$.

crecimiento fue calculada según lo citado en la bibliografía ${ }^{11}$.

El momento de la ovulación se definió como el promedio entre la última observación del folículo dominante y el momento en que éste desapareció ${ }^{15}$. El diámetro del folículo ovulatorio fue aquel determinado previamente a su desaparición. La ovulación fue confirmada cuando se detectó un cuerpo lúteo en el lugar donde había desaparecido el folículo dominante ${ }^{20}$.

Análisis estadístico. Las variables analizadas fueron sometidas al test de Kolmogorov-Smirnov para determinar normalidad de los residuos y examinadas por analisis de varianza (ANOVA), utilizando el programa estadístico SAS (PROC GLM ${ }^{24}$ ) y sometidas al test de Bartlett (realizándose tranformaciones cuando fueran necesarias), para determinar homogeneidad de varianza.

Tales variables fueron: diámetro del folículo dominante en el momento de retirar el dispositivo intravaginal, $24 \mathrm{~h}$ después y en el momento de la ovulación, tasa de crecimiento folicular, intervalo entre el retiro del dispositivo y la ovulación, y área del cuerpo lúteo.

Las concentraciones plasmáticas de progesterona y cortisol del día 0 al 12, y el día 22 , fueron analizadas por ANOVA para medidas repetidas en el tiempo (PROC MIXED ${ }^{24}$ ). La tasa de ovulación fue analizada utilizando el PROC CATMOD ${ }^{24}$. El modelo de análisis estadístico incluyó los efectos del tratamiento. Para el nivel de cortisol, el modelo incluyó la respuesta ovulatoria. Todos los valores fueron expresados como media $\pm \mathrm{EE}$.

\section{RESULTADOS}

Dinámica folicular. Se observó el surgimiento de una nueva onda de crecimiento folicular en el 83,3\% de los animales (25/30), comenzando a los $3,9 \pm 1,3$ días luego de colocado el dispositivo intravaginal. El resto de los animales tuvo un folículo dominante (FD) persistente.

Los diámetros del FD en el momento de retirar el dispositivo, a las $24 \mathrm{~h}$ posteriores y a la ovulación, no difirieron entre tratamientos ( $p>0,05$; Tabla 1 ). Sin embargo, hubo diferencias en la tasa de crecimiento del folículo ovulatorio $(p<0,05$; Tabla 1$)$.

El porcentaje de vacas que ovularon $(66,7 \% ; 20$ de $30, p>0,05)$ y el intervalo del retiro del dispositivo a la ovulación $(71,4 \pm 9,9$ h) no difirieron entre tratamientos. El diámetro del folículo dominante en el momento de retirar el dispositivo no difirió entre las vacas que ovularon y las que no lo hicieron $(11,1 \pm \mathbf{1 , 7}$ y $10,3 \pm 1,1 \mathrm{~mm}$ respectivamente, $p>0,05$ ).

En los tratamientos T1 y T1,3, el $100 \%$ de las ovulaciones se produjeron de un folículo dominante surgido de una nueva onda de crecimiento folicular; no así en el T0,558 en el que solo ocurrió en el $50 \%(\mathrm{p}<0,05)$.

Niveles plasmáticos de progesterona y cortisol, así como área del cuerpo lúteo. No se observaron diferencias en los niveles de progesterona entre los diferentes tratamientos utilizados ( $>>0,05$; Figura 1). En el día 0 , el $93,3 \%$ de las vacas tuvieron un cuerpo lúteo, los cuales fueron similares entre tratamientos $(\mathrm{T} 0,558 \mathrm{~g}=$ $310,2 \pm 129,7 ; \quad \mathrm{T} 1 \mathrm{~g}=371,4 \pm 93,8$ y $1,3 \mathrm{~g}=360,9 \pm 84,5$ $\left.\mathrm{mm}^{2} ; \mathrm{p}>0,05\right)$.

En las vacas que ovularon en el día 22, tanto el nivel de progesterona $(7,2 \pm 3,8 \mathrm{ng} / \mathrm{ml})$ como el área del cuerpo lúteo $\left(290,8 \pm 118 \mathrm{~mm}^{2}\right)$ no difirieron entre tratamientos $(p>0,05)$. Aquellas vacas que no ovularon presentaron concentraciones de progesterona inferiores a $1 \mathrm{ng} / \mathrm{ml}$.

Los niveles plasmáticos de cortisol fueron mayores en los días 8 y 9 en las vacas que no ovularon $(\mathbf{p}<0,05)$; siendo significativa la interacción respuesta ovulatoria por día del tratamiento ( $p<0,05$; Figura 2$)$. 


\section{DISCUSIÓN}

La emergencia de una nueva onda de crecimiento folicular fue observada en el $\mathbf{8 3 , 3 \%}$ de las vacas coincidiendo con lo informado por la bibliografía ${ }^{1}$, cuyos autores registraron un valor del $87,5 \%$. El rango en que surge la nueva onda de crecimiento folicular luego de administrar benzoato, es de 3 a 6 días ${ }^{4,21}$; siendo el promedio observado en el presente trabajo de 3,9 días.

La cantidad de progesterona utilizada en los dispositivos (desde $0,558 \mathrm{~g}$ hasta $1,3 \mathrm{~g}$ ) no afectó el diámetro del folículo dominante ovulatorio ni el porcentaje de ovulación; sin embargo, cabe señalar que en aquellas vacas que recibieron $0,558 \mathrm{~g}$ de progesterona, el $33,3 \%$ de las ovulaciones se originaron de un folículo persistente comparado con el $14,7 \%$ y $0 \%$ para las que recibieron $1 \mathrm{~g}$ y $1,3 \mathrm{~g}$ de progesterona.

No obstante, los niveles plasmáticos de dicha hormona no difirieron entre tratamientos, lo cual hace difícil encontrar una explicación a esta observación. Futuros trabajos deberán confirmar tal hallazgo.

El porcentaje de vacas que ovularon, independientemente del tratamiento, fue del 66,7\%. Este porcentaje es inferior al registrado en la literatura ${ }^{17}$. Los animales utilizados en el presente trabajo pertenecían a un

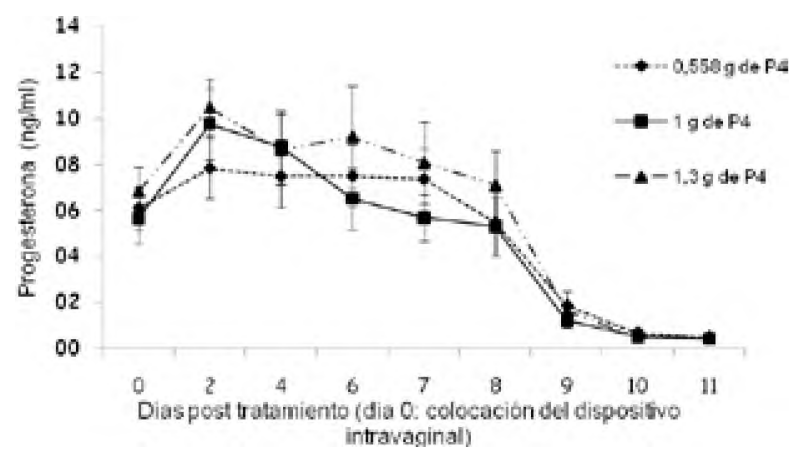

Figura 1. Niveles plasmáticos de progesterona en vacas tratadas con dispositivos intravaginales que contenían diferentes cantidades de progesterona (P4).

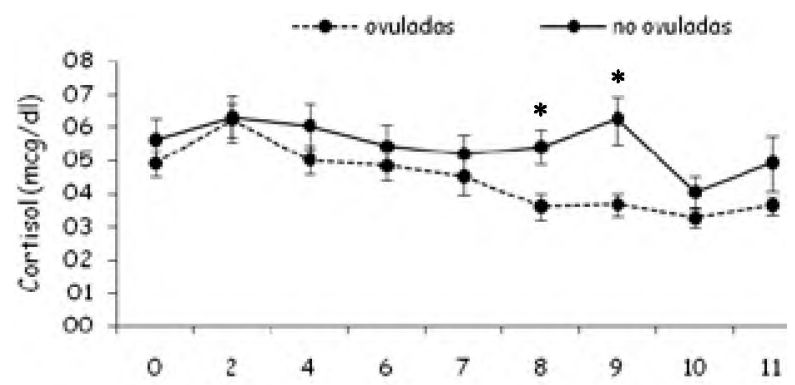

Dias post trafamiento (Oía 0 : colocaciondel dispositiwa introuginal)

Figura 2. Niveles plasmáticos de cortisol en vacas tratadas con dispositivos intravaginales con diferentes cantidades de progesterona según ovularon o no en respuesta al tratamiento. El asterisco indica diferencias significativas $(p<0,05)$ entre vacas que ovularon $y$ aquéllas que no lo hicieron. rodeo comercial y fueron sometidos a manejos muy intensivos (ecografía y sangrados diarios) que podrían haber generado estrés, explicando el menor porcentaje de ovulación.

En este sentido, se argumentó que cualquier manejo que provoque estrés en los animales genera un aumento en el nivel de glucocorticoides y podría interferir con la actividad reproductiva ${ }^{19}$. En ratas, investigadores concluyeron que bajo las condiciones en las que realizaron sus experimentos, la dexametasona podría bloquear la ovulación por inhibir la síntesis y liberación de LH, lo cual provocaría ausencia del pico preovulatorio de dicha hormona ${ }^{2}$.

En el presente trabajo se observó que los animales que no ovularon tuvieron niveles de cortisol en plasma superiores a aquellos que ovularon en los días 8 (retiro del dispositivo) y 9 (administración de BE para inducir el pico preovulatorio de LH). En consecuencia, se podría hipotetizar que el aumento de cortisol en estos animales podría haber bloqueado la generación del pico de LH, paso necesario para que los animales ovulen.

Por otro lado, en la especie porcina se ha sugerido que existe una interacción entre el status social de los animales y la respuesta al estrés. Asi, hay animales más sumisos que serían más sensibles, por lo cual serían más afectados por situaciones de estrés ${ }^{18}$. Otros autores han propuesto una situación similar en la especie bovina, en la cual habría relación entre el comportamiento individual y la respuesta al agente estresante ${ }^{12}$. Ello explicaría porqué no todas las vacas sufrieron un bloqueo de la ovulación a pesar de haber sido sometidas al mismo manejo.

Otros trabajos han estudiado la relación entre el manejo de los animales, el nivel de cortisol en plasma y la eficiencia reproductiva. Así, un investigador ${ }^{8}$, observó un aumento significativo en el nivel de cortisol (de 3,67 a 4,84 ng/ml; $\mathrm{p}<0,05$ ) en novillos que fueron manejados en forma estresante (perros, picanas, etc.) comparado con aquellos en los que se tuvieron en cuenta las normas de bienestar animal. En este sentido, se ha observado cómo un manejo inadecuado de los animales afecta negativamente la proporción de hembras que quedan preñadas luego de realizar una IATF ${ }^{26,27}$.

El diámetro del folículo dominante en el momento de retirar el dispositivo fue ligeramente superior al obtenido por investigadores que registraron valores de $9,2 \pm 0,9 \mathrm{~mm}^{23}$. A las $24 \mathrm{~h}$ posteriores el diámetro fue similar. Otros autores han informado un diámetro de $8,5 \mathrm{~mm}$ para vacas cíclicas ${ }^{16}$. Diferencias en el tipo de dispositivo utilizado podrían haber generado niveles de progesterona distintos, que podrían explicar las diferencias observadas.

Otro factor que influye en el diámetro del folículo al momento de retirar el dispositivo es el tiempo de permanencia del mismo en la vagina. En el trabajo realizado por otros investigadores, los dispositivos estuvieron colocados durante 8 días ${ }^{23}$. En el presente trabajo estuvieron un día más, lo cual hace suponer que el folículo tuvo un día más para crecer, por lo cual sería de mayor 
tamaño. El diámetro del FD ovulatorio fue coincidente con lo citado en la bibliografía ${ }^{5,6,16,23}$.

Los niveles plasmáticos de progesterona observados en el presente trabajo en aquellas vacas que ovularon, sumado a las características ováricas (porcentaje de ovulación, intervalo de retiro del dispositivo-ovulación, diámetro del FD ovulatorio) brindan las bases fisiológicas que permiten explicar la ausencia de diferencias significativas en el porcentaje de preñez que se obtiene al utilizar dispositivos con diferentes cantidades de progesterona.

Finalmente, este trabajo muestra que hay una relación entre los niveles de cortisol (en el momento en que se estimula y debería producirse el pico preovulatorio de $\mathrm{LH}$ ) y la respuesta ovulatoria. Esto podría ser un indicador de cómo fueron manejados los animales (situaciones de estrés). Tal hecho asume importancia al momento de decidir cómo manipular animales, ya que un mal manejo afectaría su eficiencia reproductiva.

Se concluye que los dispositivos intravaginales de $0,558 \mathrm{~g}$, los de $1 \mathrm{~g}$ y los de $1,3 \mathrm{~g}$ de progesterona, generan similares niveles plasmáticos de dicha hormona y cambios ováricos. Los animales que no ovulan, tienen mayores niveles de cortisol que aquellos que sí lo hacen, pudiendo esto ser un indicador de situaciones de estrés; el cual podría afectar la eficiencia reproductiva.

\section{REFERENCIAS}

1. Avilés M et al. 2005. Efecto del uso de DIB sobre el momento de inicio de onda y ovulación en vacas y vaquillonas tratadas con benzoato de estradiol. VII Simposio Internacional de Reproducción Animal, Córdoba (Argentina), p. 383.

2. Baldwin DM, Sawyer HS. 2011. Effects of dexametasone on $\mathrm{LH}$ release and ovulation in the cyclic rat. Endocrinology 94: 1397-1403.

3. Bó GA, Baruselli PS, Mapletoft RJ. 2013. Synchronization techniques to increase the utilization of artificial insemination in beef and dairy cattle. Anim Reprod 10: 137142.

4. Burke CR, Day ML, Bunt CR, McMillan KL. 2000 . Use of a small dose of oestradiol benzoate during diestrus to synchronize development of the ovulatory follicle in cattle. J Anim Sci 78: 145-151.

5. Cavalieri J, Hepworth G, Parker KI, Wright PJ, McMillan KL. 2003. Effect of treatment with progesterone and oestradiol when starting treatment with an intravaginal progesterone releasing insert on ovarian follicular development and hormonal concentrations in Holstein cows. Anim Reprod Sci 76: 177-193.

6. Cavalieri J, Hepworth G, McMillan KL. 2004. Ovarian follicular development in Holstein cows following synchronization of oestrus with oestradiol benzoate and an intravaginal progesterone releasing insert for 5-9 days and duration of the oestrous cycle and concentrations of progesterone following ovulation. Anim Reprod Sci 81: 177-193.
7. Confalonieri OE et al. 2006. Model of hyperalgesia associated with lameness in dairy cattle. J Anim Vet $A d v 5$ : 1214-1219.

8. Costa A, Dasso L. 2007. Manejo de bovinos en sistemas productivos: caracterización de dos estilos de manejo y niveles sanguíneos de cortisol. Prod Syst in Bov Manag., Red Vet (http://www.veterinaria.org/revistas/redvet/ n121207B.pdf.)

9. Fike KE et al. 1997. Estrus, ovulation, and luteal function in anestrous suckled beef cows given an implant of progesterone with or without a subsequent injection of estradiol. J Anim Sci 75: 2009-2015.

10. Ginther OJ, Knopf L, Kastelic JP. 1989a. Temporal association among ovarian events in cattle during oestrus cycles with two and three follicular waves? $J$ Reprod Fert 87: 223-230.

11. Ginther OJ, Kastelic JP, Knopf L. 1989b. Composition and characteristics of follicular waves during the bovine estrous cycle. Anim Reprod Sci 20: 187-200.

12. Gonzalez M, Yabuta AK, Galindo F. 2003. Behaviour and adrenal activity of first parturition and multiparous cows under a competitive situation. Applied Anim Behav Sci 83: 259-266.

13. Houghton PL, Lemenager RP, Moss GE, Hendrix KS. 1990. Prediction of postpartum beef cow body composition using weight to height ratio and visual body condition score. J Anim Sci 68: 1428-1437.

14. Lane EA, Austin EJ, Crowe MA. 2008. Oestrus synchronization in cattle. Current options following the EU regulations restricting the use of oestrogenic compounds in food-producing animals. Anim Reprod Sci 109: 1-16.

15. Lemaster JW, Yelich JV, Kempfer JR, Schrick FN. 1999. Ovulation and estrus characteristics in crossbred Brahman heifers treated with an intravaginal progesterone-releasing insert in combination with prostaglandin F2 alpha and estradiol benzoate. J Anim Sci 77: 1860-1868.

16. Manes $\mathbf{J}$ et al. 2008. Follicular dynamic in postpartum anoestrous and cyclic cows treated with medroxi-progesterone acetate (MPA) and estradiol benzoate (EB). Reprod Domest Anim 43: 172-173.

17. Mapletoft RJ, Martínez MF, Colazo MG, Kastelic JP. 2003. The use of controlled internal drug release devices for the regulation of bovine reproduction. J Anim Sci 81 (Suppl. 2): E28-E36.

18. McGlone JJ et al. 1993. Shipping stress and social status effects on pig performance, plasma cortisol, natural killer cell activity, and leukocyte numbers. J Anim Sci 71: 888896

19. Moberg G. 1976. Effects of environment and management stress on reproduction in the cow. J Dairy Sci 59: 16181624.

20. Murphy MG, Boland MP, Roche JF. 1990. Pattern of follicular growth and resumption of ovarian activity in post-partum beef suckler cows. J Reprod Fert 90: 523-533.

21. O'Rourke M, Diskin M, Greenan JM, Roche JF. 2000. The effect of dose and method of oestradiol administration on plasma concentrations of oestradiol and FSH in longterm ovariectomised heifers. Anim Reprod Sci 59: 1-12. 
22. Rogan D et al. 2007. Progesterone release patterns from cue-mate in comparison to other intravaginal progesterone-releasing devices in lactating dairy cows. Reprod Fertil \& Develop 19: 126-127.

23. Ross PJ et al. 2004. Estradiol benzoate given 0 or $24 \mathrm{~h}$ after the end of a progestagen treatment in post-partum suckled beef cows. Theriogenology 62: 265-273.

24. SAS/STAT Foundation (Statistical Analysis Software Institute Inc.). 1989. User's Guide, version 6, Fourth ed., vol. 2, Cary NC (USA), p. 846.

25. Toribio RE, Molina JR, Bolaños JM, Kindahl H. 1994. Blood levels of the prostaglandin F2 $\alpha$ metabolite during the post-partum period in Bos indicus cows in the humid tropics. J Vet Med A-41, 630-639.
26. Vater A et al. 2008. Dos formas de manejo de vaquillonas sometidas a inseminación a tiempo fijo (IATF) y sus efectos sobre la tasa de preñez. Rev Arg ProdAnim 28 (Supl. 1), p. 148-149.

27. Vater A et al. 2009. Efecto del manejo de vacas con cría durante la implementación de una IATF sobre la tasa de preñez. XXXVII Jornadas Uruguayas de Buiatria, Uruguay, p. 138-139

\section{SClmago Journal \& Country Rank}

\section{Revista Veterinaria mantiene su índice de impacto}

Noticias de Scimago Research Group (Scimago Journals \& Country Ranks, Scopus-Elsevier) comunican que la publicación de nuestra casa, Revista Veterinaria, aumentó su índice de impacto. El índice SJR mide la influencia científica (impacto) del artículo de una revista, expresando cuán importante es el "artículo promedio" de la publicación en la discusión científica global (sistema Thomson Reuters).

Para nuestra revista, tal indicador había sido de 0,03 entre 2008 y 2011, aumentando a 0,05 en 2012, a 0,11 en 2013, a 0,108 en 2015, a 0,100 en 2017 y a 0,114 (Research Gate) en 2018, último período evaluado. Asimismo, surge para nuestra publicación un sostenido descenso del indicador que relaciona "citas versus autocitas", demostrando que los autores de los artículos están abandonando la práctica de citar sus propias publicaciones anteriores.

Por último, se advierte que según este portal, nuestra "Revista Veterinaria" continúa siendo la única publicación de esta rama de la ciencia que posee índice de impacto en Argentina. Para el resto del cono sur tal distinción recae en Brasil, Chile, Colombia y Venezuela. En revistas de veterinaria, a nivel mundial el mayor índice de impacto (2,911 puntos) recae en Annual Review of Animal Biosciences (Estados Unidos). 